\title{
Mediaciones parentales y uso de internet por niños, niñas y adolescentes colombianos
}

\section{Parental mediations and internet use by colombian children and adolescents}

\author{
Norman Darío Moreno-Carmona ${ }^{1}$, Andrés Marín-Cortés ${ }^{2}$, \\ Víctor Hugo Cano-Bedoya ${ }^{3}$, Jorge Armando Sanabria-González \\ Ángela María Jaramillo-Suarez ${ }^{5}$ y Julio César Ossa-Ossa ${ }^{6}$
}
'Psicólogo. Magíster en Educación: Desarrollo Humano. Doctor en Investigación Psicológica.
Profesor Titular de la Facultad de Psicología, Universidad de San Buenaventura, Medellín, Colombia. E-mail: norman.moreno@usbmed.edu.co
${ }^{2}$ Psicólogo y Magíster en Psicología. Profesor Asociado de la Facultad de Psicología,
Universidad de San Buenaventura, Medellín. E-mail: andres.marin@usbmed.edu.co
${ }^{3}$ Psicólogo. Magíster en Psicología. Jefe del CIAF y Psicólogo de Bienestar Institucional, Universidad de San Buenaventura, Medellín, Colombia. E-mail: victor.cano@usbmed.edu.co ${ }^{4}$ Psicólogo. Magíster en Pensamiento Complejo. Docente de tiempo completo en la Facultad de
Psicología, Universidad de San Buenaventura, Medellín, extensión Ibagué, Colombia.
E-mail: jorge.sanabria@usbmed.edu.co

${ }^{5}$ Psicóloga, Magíster en Psicología Educativa. Docente de tiempo completo en la Facultad de

Ciencias Humanas y Sociales, Universidad de San Buenaventura, Cali, Colombia.

E-mail: amjaramillo01@usbcali.edu.co

${ }^{6}$ Psicólogo. Magíster y Doctor en Psicología. Al momento de realizar la investigación se desempeñaba como Director de Investigaciones y Editor de la Revista Guillermo de Ockham,

Universidad de San Buenaventura, Cali, Colombia. E-mail: juceossa@gmail.edu.co

La investigación que se presenta fue financiada por la Universidad de San Buenaventura, seccionales Cali y Medellín, y realizada en colegios públicos y privados de las ciudades de Medellín (Antonio Derka, Alcaldía de Medellín, Gilberto Alzate Avendaño, Javiera Londoño, Colegio Pestalozzi y The New School), Cali (General Francisco de Paula Santander, Colegio IETI, El Carmelo, IE Multipropósito, Liceo Galeón, Los Ángeles de San Fernando, Instituto Comercial Arquidiocesano), Pasto (Liceo José Félix Jiménez, IEM San Juan Bosco e IEM Libertad), Ibagué (Normal Superior, Simón Bolívar, Sagrada Familia y San Bonifacio de las Lanzas), Bello (Josefa Campos), Palmira (Comfandi y Seminario de Cristo Sacerdote), Tumaco (General Santander y Nuestra Señora de las Lajas), Santander de Quilichao (Francisco José de Caldas e Instituto Técnico), Támesis (IE Agrícola Víctor Manuel Orozcoy San Antonio de Padua) y Espinal (Colegio Mariano Sánchez), de Colombia.

\section{Resumen}

Este artículo presenta los resultados de un estudio correlacional sobre los controles y las mediaciones parentales frente al uso de internet por niños, niñas y adolescentes colombianos. Se trata de la encuesta EU Kids Online que fue aplicada en el año 2010 en 25 países europeos $\mathrm{y}$, posteriormente, en Brasil en 2013 y en Chile en 2016. En el presente estudio, le encuesta 
fue adaptada y validada para la población colombiana mediante un panel de expertos y entrevistas cognitivas a usuarios. Se conformó una muestra estratificada y no probabilística de 303 estudiantes de ambos sexos de colegios públicos y privados, con edades entre 9 y 17 años y uno de sus padres o cuidadores, habitantes de 11 ciudades del país, quienes respondieron a los tres instrumentos de Kids Online, una encuesta autoaplicada, otra dirigida a los estudiantes, y una tercera dirigida a los padres o cuidadores. Los resultados muestran relaciones entre el control parental y un menor tiempo de conexión a internet por parte de los hijos: el control del padre está relacionado con menor presencia de conductas adictivas a internet y al celular y, por el contrario, una relación autoritaria con la madre está relacionada con mayor presencia de conductas de riesgo en los hijos y específicamente, en el uso de internet. Además, las habilidades digitales de los padres son las que mejor están asociadas con una mediación activa, que los involucra más en las actividades digitales de sus hijos.

Palabras clave: infancia, adolescencia, mediaciones parentales, uso de internet, conductas de riesgo.

\section{Abstract}

Nowadays, parents must develop strategies to accompany their children in the use of digital technologies. The reason for this is that the internet is a social space. Online life offers possibilities for individual and social development, but it also promotes the emergence of new risks such as sexting, cyberbullying, and cyber dependence. There are two ways parents can guide their children: parental mediation and parental control. Parental mediation is the set of actions that parents take to manage the use and consumption of the media by their sons and daughters. There are three types of parental mediation: restrictive mediation, permissive mediation, and active mediation. In this context, this research aims to correlate mediation and parental control strategies with their use of the internet for children and adolescents. The EU Kids Online survey was used to achieve this goal. This questionnaire was adapted for the Colombian population through expert review and cognitive validation to children and adolescents in Medellin, Cali, and Cartagena (Colombia). The adaptation followed the procedures and standards of Kids Online Brasil and Kids Online Chile. The stratified and non-probabilistic sample consisted of 303 children and adolescents aged 9 to 17 years from ten cities in Colombia. The results indicate that the more parental control, the shorter the child connection time. Whether it's behavioral or psychological control. While the greater the psychological control of the mother, the greater the presence of addictive behaviors of children and adolescents to the internet and cell phone. Besides, the more psychological control of the mother, the more risky behaviors the children assume. Conversely, if the mother performs greater behavioral control, the presence of risky behaviors in the child is less. Also, restrictive control of internet use has a higher relationship with a lower presence of general risk behaviors and lower risks assumed by children on the internet. This was also related to high rates of restrictive mediation by parents. As conclusions, it is possible to say that any type of control that parents exercise over their children's use of the internet (restrictive, co-visioned or technical) is related to shorter connection time and less cell phone addiction. Besides, a conflicting relationship with the mother increases the addictive behaviors of the internet and the smartphone. Especially when the kind of control the mother exerts is psychological. Whereas, if the mother exerts greater behavioral control, the presence of risky behaviors in the child is less. This research could serve as the basis for the design and implementation of psychoeducational training programs for parents and caregivers aimed at developing effective parental mediation skills and strate- 
gies to guide children and adolescents in their use of digital technologies. Also, the findings of this study could lay the scientific basis for the presentation of social projects that impact public policies on the responsible and healthy use of the internet. Moreover, when Colombia has a Ministry of Information and Communication Technologies and media outreach strategies on ICT care. Finally, researches done in psychology about this topic are limited in Latin America. For this reason, the findings of this work are a significant step forward on the explanation of ITC use in the region from a disciplinary point of view. This article seeks to encourage researchers to continue to advance their knowledge of the relationships between parental mediation and the use of the internet by children and adolescents.

Keywords: childhood, adolescence, parental mediation, internet use, risky behaviors.

\section{Introducción}

En la actualidad, los niños, las niñas y los adolescentes (NNA) utilizan sus smartphones y tablets el doble del tiempo en comparación con el uso que hacen de las computadoras (Chang et al., 2018). Esta situación amerita que los padres, las madres y los cuidadores desarrollen habilidades y estrategias para acompañar a los menores de edad en el uso que hacen de internet y las tecnologías digitales. Sobre todo, porque el mundo de hoy invita a la interacción digital y a la conectividad permanente. En este contexto, este trabajo de investigación se ocupa de las relaciones entre la mediación parental, el control parental y el uso de internet por parte de NNA.

La mediación parental es importante porque puede afectar las maneras en las que los NNA utilizan la tecnología. Este concepto hace referencia al conjunto de acciones que llevan a cabo los padres y madres para gestionar el uso y consumo de los medios de comunicación por parte de sus hijos e hijas (Livingstone y Helsper, 2008). La mediación parental también está centrada en el tiempo que los NNA pasan en medios digitales (Garmendia, Jiménez, Casado y Mascheroni, 2016).

Existen tres tipos de mediación parental: la mediación restrictiva, la mediación permisiva y la mediación activa. La primera de ellas se refiere a los métodos utilizados por los padres y madres para restringir el acceso de sus hijos a los contenidos y medios digitales (Yubero, Larrañaga, Navarro y Elche, 2018). Este tipo de mediación puede manifestarse estableciendo límites de tiempo de uso, restringiendo el acceso a los dispositivos móviles, instalando filtros de internet o controlando la selección de los contenidos. El segundo tipo de mediación es la permisiva, la cual se entiende como la ausencia de monitorización del uso que los menores de edad hacen de las Tecnologías de la Información y la Comunicación (TIC) (Özgür, 2016). Finalmente, la mediación activa se presenta cuando los padres participan de las experiencias digitales de sus hijos a través de conversaciones y apoyo cognitivo y social. Este tipo de mediación suele desarrollar el pensamiento crítico en los NNA, así como habilidades sociales para las interacciones cara a cara (Uhls y Robb, 2017).

La mediación parental es uno de los factores ambientales más importantes para el desarrollo de los NNA, incluyendo su bienestar psicológico y rendimiento académico. Vargas- $\mathrm{Ru}-$ bilar y Arán-Filippetti (2014) sostienen que el acompañamiento permanente de los padres y las madres cumple un rol relevante en el desarrollo cognitivo de los niños y niñas. Específicamente, la mediación tiene una incidencia positiva en las funciones ejecutivas, la memoria de trabajo, la flexibilidad cognitiva y la inhibición conductual. Por otra parte, Suárez y Vélez (2018) indican que los estilos parentales favorecen el desarrollo de habilidades sociales en sus hijos, y contribuyen a las capacidades para relacionarse con los demás de forma empática.

Por su parte, el control parental involucra la regulación de las actividades y rutinas de los menores de edad, así como el establecimiento de reglas y restricciones. Adicionalmente, puede ser conceptualizado como control psico- 
lógico y control conductual. El primer tipo se refiere al intento por controlarlas emociones y las creencias de los hijos, limitando su autonomía psicológica y utilizando estrategias como las críticas excesivas, el afecto condicionado, la inducción de culpa, la comunicación restrictiva, la invalidación de sentimientos, la sobreprotección y la intrusividad (Moreno Carmona, 2018).El segundo tipo, el control conductual, involucra la regulación de las actividades y rutinas de los hijos a través del conocimiento que los padres tienen de ellas (Wong, Zhuang y Ng, 2018). Este control se refiere a los intentos de los padres por regular, supervisar y controlar la conducta de los NNA, de acuerdo con las normas familiares o sociales (Baumrind, 2005).

De acuerdo con Yubero, Larrañaga, Navarro y Elche (2018), los padres que no utilizan habitualmente internet suelen sentir más preocupación respecto al uso que sus hijos hacen de dicha tecnología, en comparación con aquellos que lo usan regularmente. Sin embargo, en la medida en que los hijos crecen, dicha preocupación va disminuyendo. Cuando los padres y las madres son más activos en internet suelen emplear más estrategias de mediación activa, que restrictiva. A pesar de los prejuicios relacionados con el uso de internet y el consumo de contenidos digitales, los padres, las madres y los cuidadores aceptan que, para sus hijos, usar internet es una actividad indispensable (Pichardo, Fernández, García y García, 2014).

Según lo encontrado por Warren y Aloia (2019) y Festl y Gniewosz (2018), la educación de los padres está relacionada con las estrategias de mediación del uso de smartphones en sus hijos. Entre más altos son los niveles educativos de los adultos, mayor es el nivel de mediación de las tecnologías de la información y la comunicación (TIC). Además, los padres con niveles educativos más altos tienen un estilo parental que demuestra mayor confianza. En esta misma línea, Jiménez-Morales, Montaña y Medina-Bravo (2020) encontraron que los hijos cuyas madres tienen menor formación académica tienen un mayor consumo de contenidos digitales. En cuanto a los estilos parentales, un estilo parental autoritario está positivamente relacionado con una mediación restrictiva del uso de celular. Mientras que un estilo parental permisivo está positivamente relacionado con una mediación covisionada del uso del celular (Warren y Aloia, 2019; Festl y Gniewosz, 2018).

No obstante, diversas investigaciones en Europa y Latinoamérica han encontrado predominancia de la mediación restrictiva (Garmendia, Casado del Río y Martínez, 2016; Martínez de Morentin, Cortés y Medrano, 2014; Torrecillas-Lacave, Vázquez-Barrio y Monteagudo, 2017). Además, Wright (2016) afirma que la mediación parental restrictiva está positivamente relacionada con el cyberbullying, mientras que este tiene una relación negativa con la mediación instructiva y covisionada. Por otro lado, la comunicación abierta con los cuidadores y padres, en particular el apoyo materno y las demostraciones de cariño constituyen factores protectores frente a la cibervictimización (Marín-Cortés, Hoyos de los Ríos y Sierra, 2018).

El uso extendido de tecnologías digitales ha generado diversas problemáticas sociales propias de los entornos online, las cuales están relacionadas con el tipo de mediación que establecen los padres, madres y cuidadores. De acuerdo con Tavares, Falcke y Pereira (2019), una práctica común entre los adolescentes es el sexting. En su investigación, se encontró que los padres sienten mayor preocupación por el hecho de que los contenidos se hagan virales, que por la práctica en sí misma. Según los padres, lo que motiva a sus hijos a realizar sexting es la curiosidad y el propósito de impresionar positivamente a los demás en social media (medios sociales). Aunque, en general, los adolescentes conocen los riesgos relacionados con esta práctica, consideran que es una forma común para establecer vínculos sexoafectivos. Además, los jóvenes creen que las consecuencias del sexting son más negativas para las mujeres que para los varones (Alonso-Ruido, Rodríguez-Castro, Lameiras-Fer- 
nández y Martínez-Román, 2018).

Otra de las problemáticas asociadas al uso de tecnologías digitales por parte de los menores de edad es la adicción a internet. Los adolescentes pertenecientes a niveles medio y con disfuncionalidad familiar, de familias de entre uno a tres miembros, tienen mayor probabilidad de desarrollar adicción a internet. Además, los jóvenes provenientes de familias con condiciones económicas de clase media reportaron una prevalencia más alta de ciberdependencia (Aponte, Castillo y González, 2017).

En cuanto al cyberbullying, Álvarez-García, Núñez, García y Barreiro-Collazo (2018) encontraron que variables familiares como el control conductual, las reglas en el uso de internet, la monitorización en el uso de las TIC y la comunicación operan como factores protectores. Además, el estilo parental influye en la empatía y la impulsividad de los adolescentes. Cuando los padres tienen estilos parentales controladores, sus hijos reportan niveles más elevados en involucramiento de cyberbullying, tanto como perpetradores y como víctimas. Además, los padres que son inconsistentes en su estilo de mediación cibernética, por ejemplo, estableciendo reglas sin reforzarlas ni seguir su cumplimiento, tienen hijos que se involucran en situaciones de ciberacoso (Katz, Lemish, Cohen y Arden, 2019).

Específicamente, sobre mediación parental se han llevado a cabo diversos estudios en Iberoamérica. Pérez y Torres (2020) encontraron que los padres, las madres y los docentes suelen privilegiar la mediación parental restrictiva. Los autores señalan que los adultos que llevan a cabo acciones dirigidas a estos tipos de mediación suelen hacer poco uso de internet y contar con bajas habilidades tecnológicas. Aponte, Castillo y González (2017) encontraron que, entre los factores protectores frente a la adicción a internet están el establecimiento de vínculos estrechos entre padres e hijos y las estrategias de mediación restrictivas. Sin embargo, es necesario combinar la mediación restrictiva con acciones de mediación activa (Chang et al., 2018).
En cuanto a los procesos psicosociales implicados en las relaciones entre el uso de internet por parte de NNA y la mediación y estilo parental, Festl y Gniewosz (2018) encontraron que la confianza percibida por los padres y las madres es el recurso más importante para la cohesión familiar. Aquellos con mayor confianza se involucran más en el uso de internet de sus hijos, y, al mismo tiempo, la confianza está relacionada con un clima familiar más positivo. Respecto a los miembros familiares que supervisan el uso que hacen de internet los menores de edad, en primer lugar, están los padres y las madres, seguidos por los hermanos mayores y, finalmente, los abuelos. En cuanto a las diferencias de género, las niñas son más controladas por los adultos, en comparación con los niños (Arnaiz, Cerezo, Giménez y Maquilón, 2016).

Bartau-Rojas, Aierbe-Barandiaran y Oregui-González (2018) se propusieron investigar sobre las creencias, las dificultades y las prácticas de los padres y las madres para enseñar a sus hijos sobre los riesgos y beneficios del uso de internet. En su estudio, los autores encontraron que los adultos utilizan diversas estrategias de mediación parental restrictiva, tales como establecer normas, límites y supervisión del uso de las TIC. Además, los padres y madres emplean diversas estrategias de mediación activa, como es el caso de la comunicación y la instrucción, que hacen parte de su estilo de parentalidad más amplio. Es decir, es un estilo que se lleva a cabo más allá del uso de las tecnologías digitales.

Por su parte, en Colombia se han realizado pocas investigaciones sobre mediación parental y uso de tecnologías digitales por parte de NNA. En la revisión de antecedentes, únicamente se encontró la investigación llevada a cabo por Osorio-Tamayo y Millán (2020), quienes encontraron que los adolescentes consideran que sus padres y madres alternan entre la mediación permisiva y el control excesivo. Además, algunos cuidadores justifican su desentendimiento sobre las actividades que realizan sus hijos diciendo que únicamente 
utilizan internet para realizar sus actividades académicas.

Para el presente estudio se diferenció el control como práctica parental general del control que ejercen sobre el uso de internet y que es percibido por los hijos como covisionado, restrictivo y/o técnico. Además, se relacionó la mediación parental reportada por los padres como activa, restrictiva y/o técnica con el involucramiento de los NNA colombianos en algunas conductas de riesgo.

En el marco de todo lo anterior, esta investigación es importante porque el uso que los NNA hagan de las tecnologías digitales, sobre todo a edades tempranas, puede determinar la manera en la que afrontarán las situaciones sociales que en ella se presentan. El sexting, el cyberbullying y la ciberdependencia no se tratan únicamente de la manera en que se utilizan los dispositivos de pantalla, sino además de las interpretaciones que las personas hacen de las relaciones con ellas mediadas por la tecnología. Enfocarse en las estrategias de mediación y de control parental respecto al uso de las TIC puede favorecer la comprensión sobre las actividades y representaciones que tienen los menores de edad sobre los social media, los videojuegos, los contenidos digitales, etcétera. Además, esta investigación es relevante en Colombia debido a que es muy limitado el conocimiento producido en el país respecto a las relaciones entre la mediación parental y el uso de internet por parte de las generaciones más jóvenes.

De acuerdo con este marco, la pregunta que orientará el trabajo será por las relaciones de los controles y las mediaciones parentales con el uso de internet en niños, niñas y adolescentes colombianos. Y a partir de allí, las hipótesis del estudio plantean que existe relación entre el tipo de control de los padres tanto con el tiempo de conexión de los estudiantes a internet, como con sus conductas de riesgo. También se analiza que existe relación entre el tipo de mediación que reportan los padres y sus habilidades digitales en internet $y$, finalmente, que existe una relación entre la mediación de los padres y el tipo de control percibido por los hijos.

\section{Método}

Luego de la adaptación y validación para Colombia (ver Procedimiento) de la encuesta EU Kids Online que fue aplicada el año 2010 en 25 países europeos y, posteriormente, en Brasil en el año 2013 y en Chile en el 2016, se realizó un estudio correlacional de los controles y las mediaciones parentales sobre el uso de internet por parte de los NNA colombianos.

\section{Muestra}

La muestra es estratificada, no probabilística, compuesta por 303 niños, niñas y adolescentes de 9 a 17 años, de los cuales el 20.3 $\%$ tenía entre 9 y 10 años y el $53.7 \%$ eran mujeres, estudiantes de colegios públicos $\mathrm{y}$ privados de las ciudades de Medellín, Bello, Támesis, Cali, Palmira, Santander de Quilichao, Pasto, Tumaco, Ibagué y Espinal. El principal criterio de inclusión fue que el niño, la niña o el adolescente fueran usuarios de internet. Se consideró la definición de usuario de internet utilizada en Kids Online Brasil y Chile, que es "una persona que ha utilizado dicha tecnología al menos una vez en los últimos tres meses".

La muestra también incluyó a uno de los padres o tutores del estudiante, generalmente quien firmaba el consentimiento informado y que tuviera algún conocimiento de las actividades del niño o adolescente en internet. El $66 \%$ de los adultos encuestados correspondió a la madre del estudiante y el $19 \%$, al padre. Los demás correspondieron a otro familiar a cargo del menor de edad.

\section{Instrumentos}

Los instrumentos que se aplicaron fueron dos cuestionarios para los niños, las niñas y los adolescentes, uno autoaplicado (172 ítems) y el otro dirigido (233 ítems). De ese cuestionario autoaplicado existen dos versiones: uno para niños y niñas de 9 y 10 años y otro para 
los de 11 a 17 años, cuya diferencia son diez preguntas específicas sobre sexting realizadas al grupo de mayor edad. También se aplicó un cuestionario dirigido a los padres o tutores de los niños (197 ítems). La encuesta dirigida por el encuestador fue la misma para todos.

Todos los cuestionarios indagan sobre usos, riesgos, mediaciones, oportunidades y datos sociodemográficos, algunos sobre la frecuencia con que le han ocurrido o ha realizado cierto tipo de eventos, mediante escalas tipo Likert con cuatro opciones de respuesta que van de nunca a siempre, muy en desacuerdo a muy de acuerdo o de falso a totalmente cierto (con valores numéricos de 1 a 4).

Las preguntas que indagan por frecuencias son del tipo: "En el último año, ¿te ha pasado alguna de las siguientes cosas?: 1) alguien usó mi información personal de una manera que no me gustó (Sí/No); 2) dejé de comer o dormir por estar conectado a internet (Sí/ No); 3) a veces uso mi celular en lugares o situaciones donde no debería (Sí/No)". Mientras que las preguntas que indagan por las mediaciones parentales fueron del tipo: ¿Tus padres (padre o madre, o la persona que te cuida) con qué frecuencia hacen las siguientes cosas?: Hablan contigo sobre lo que haces en internet; controlan el tiempo que puedes usar internet; revisan las páginas que has visitado en internet.

En el auto reporte de los niños, las niñas y los adolescentes se incluyó una escala de prácticas parentales mediadas por el celular (Zapata y Moreno, en prensa), específicamente relacionadas con control psicológico y control conductual ejercido tanto por la figura materna como por la paterna. Dicho instrumento se construyó partiendo de la Escala Prácticas Parentales en Adolescentes (PP-A) de Andrade y Betancourt (2008), adaptada para Colombia por Moreno-Carmona (2014).

\section{Procedimiento}

Para la presente investigación, el proceso de adaptación y validación de los instru- mentos se llevó a cabo mediante revisión de expertos y validación cognitiva con entrevistas a NNA y sus padres en las ciudades de Medellín, Cali y Cartagena. Para lograr esto se partió del análisis y la validación del instrumento llevado a cabo por la Pontificia Universidad Católica de Valparaíso (Chile), en el que se buscó discriminar la pertinencia de los ítems del cuestionario, así como la necesidad de reformular, descartar y conservar ítems.

Esta modalidad de trabajo buscó seguir los procedimientos y aplicar los estándares de la adaptación del proyecto europeo original y Kids Online Brasil y Chile, que permitió revisar preliminarmente la actualización de los contenidos, especialmente los referentes a tecnologías disponibles y a los nuevos usos que han surgido en los últimos años.

Una vez que se contó con el cuestionario utilizado en Chile, se procedió a su validación para el contexto colombiano. Primero se contactaron catorce expertos, entre académicos, funcionarios públicos y ONGs para invitarlos a participar en una jornada de discusión y validación, en la que se les explicaron claramente los objetivos y lo que implicaba su participación. Los instrumentos fueron distribuidos a los expertos junto a una ficha de evaluación para que revisaran y prepararan su participación en la jornada de trabajo en la que primero se presentó el estudio Kids Online y sus principales resultados en Europa, Brasil y Chile, y luego se separó a los asistentes en grupos de trabajo por temas de la encuesta. Al final de la jornada, cada grupo presentó sus análisis y principales conclusiones, y se generaron instancias de discusión entre todos los expertos.

Los aspectos a evaluar por parte de los expertos y expertas fueron: (1) pertinencia de preguntas para nivel de desarrollo psicosocial; (2) pertinencia de dimensiones exploradas para el contexto colombiano; (3) alcance y proyecciones de los datos a obtener; y (4) aspectos metodológicos generales. La validación cognitiva de los cuestionarios se llevó a cabo mediante el método de entrevistas 
cognitivas, que permiten estudiar la manera en que las personas comprenden, procesan mentalmente y responden a un instrumento, involucrando tanto procesos de pensamiento hablado y sondeo verbal (Willis, 2005). La información recogida en estas entrevistas permitió hacer modificaciones o adaptaciones a las preguntas de los cuestionarios, a partir de la manera en que los encuestados interpretaron y respondieron a las preguntas.

Se aplicaron las entrevistas cognitivas a una muestra total de 12 hogares (12 niños/ as y 12 padres/madres), de Medellín, Cali y Cartagena, considerando un balance en cuanto a edad de los niños, género, nivel socioeconómico de los padres y nivel de uso de internet de los padres (usuario/no usuario). Durante las entrevistas se buscó obtener recomendaciones específicas sobre los ítems de los instrumentos para cambios en uso de palabras, redacción, necesidad de mayor especificación, así como problemas asociados al orden, largo o carga cognitiva de las preguntas. Siguiendo a Tourangeau (1984), específicamente las dimensiones exploradas son: (1) compren- sión de la pregunta; capacidad de recordar elementos importantes para la respuesta; (2) motivaciones que orientan la toma de la decisión sobre la respuesta; y (3) mapeo de los procesos involucrados en la respuesta. Siguiendo este modelo, se plantean preguntas del tipo: “¿Puede repetir la pregunta en sus propias palabras? ¿Cómo recuerda eso? ¿Le pareció fácil o difícil esa pregunta? ¿Qué significa para usted (concepto)? ¿Cómo llegó a esa respuesta?"

Los datos del estudio se recopilaron y administraron utilizando las herramientas electrónicas de captura de datos REDCap alojadas en la página web de la Universidad de San Buenaventura Cali. REDCap (Research Electronic Data Capture) es una plataforma de software segura basada en la web diseñada para admitir la captura de datos para estudios de investigación. Los análisis de la base de datos se realizaron mediante el SPSS v.25.

En la Tabla 1 se muestra la confiabilidad (Alfa de Cronbach) obtenida por cada una de las escalas utilizadas en el análisis de las mediaciones parentales.

Tabla 1

Confiabilidad de las subescalas utilizadas.

\begin{tabular}{c|c} 
Escala (\# ítems) & Alfa de Cronbach (N) \\
\hline Control conductual materno (8) & $.910(212)$ \\
Control conductual paterno (8) & $.965(173)$ \\
Control psicológico materno (8) & $.750(215)$ \\
Control psicológico paterno (9) & $.807(176)$ \\
Control covisionado (18) & $.946(222)$ \\
Control restrictivo (6) & $.792(233)$ \\
Control técnico (7) & $.898(140)$ \\
Mediación activa (13) & $.923(224)$ \\
Mediación restrictiva (17) & $.944(165)$ \\
Mediación técnica (5) & $.805(212)$ \\
Monitoreo (4) & $.871(227)$
\end{tabular}

Fuente: elaboración propia. 
Se calculó un índice de adicción a internet a partir de la presencia de las siguientes conductas relacionadas con estar demasiado tiempo conectado a internet: dejar de comer o dormir, tener problemas con familia o amigos, bajo rendimiento académico, intentos fallidos de pasar menos tiempo conectado, problemas en general por la cantidad de tiempo que pasa conectado. También se elaboró un índice de adicción al celular, a partir de la presencia de las siguientes conductas: necesidad de revisar el celular, molestia por estar sin señal o batería, hacer cosas sin importancia en el celular, usarlo en lugares y momentos inapropiados, intentos fallidos de estar menos pendiente del celular, problemas con familia o amigos.

Asimismo, se calculó un índice de conectividad a partir de la frecuencia de conexión (desde varias veces al día hasta menos de una vez al mes), y el tiempo de conexión entre semana y los fines de semana (desde menos de media hora hasta ocho horas o más al día). Finalmente, se evaluaron las conductas de riesgo en general -como consumo de alcohol, faltar a clases, problemas con los profesores, problemas con la policía, relaciones sexuales-, así como las conductas de riesgo específicas del uso de internet -interactuar con desconocidos y enviarles información privada $\mathrm{o}$ encontrarse personalmente con ellos, fingir ser alguien diferente, ofender a otros, frecuentar contenido sexual o postearlo a otros y visitar páginas con otros contenidos no adecuados, como formas de dañarse, suicidio, violencia, sangrientas, de discriminación, uso de drogas, anorexia o bulimia.

\section{Consideraciones éticas}

La participación de los menores de edad contó con el consentimiento firmado de sus padres, por lo que la selección estuvo sujeta tanto a la obtención del consentimiento de los padres, así como a la disposición voluntaria de los niños, las niñas y los adolescentes para participar (asentimiento informado). Para el caso del adulto informante, en el consentimiento informado los padres o tutores debían aceptar su propia participación en el estudio, además de consentir la participación de su hijo. Por eso, se realizó la comunicación telefónica con cada uno de ellos, a través del número que reportaron en el consentimiento, con el fin de concretar la manera en que quería ser entrevistado: en el colegio, recibiendo al encuestador en su casa o a través de videoconferencia por internet. El consentimiento y los instrumentos tuvieron el aval del Comité de Bioética de la Universidad de San Buenaventura (Medellín), según carta de aprobación del 26 de septiembre de 2017.

\section{Resultados}

A continuación, se presentan los principales hallazgos en relación con la comprobación de las hipótesis planteadas, que se obtuvieron mediante análisis estadísticos no paramétricos, específicamente con correlación de Spearman (Rho), debido a que las variables no tuvieron una distribución normal (prueba Kolmogorov-Smirnoff).

Con respecto al control de los padres como práctica parental en general, la Tabla 2 evidencia que, a mayor control ejercido por el padre, menor será el tiempo de conexión de los hijos tanto si el control es conductual $(R h o=-.282)$ como psicológico $(R h o=$ -.306), mientras que respecto al control específico sobre el uso de internet, cualquier tipo de control que los padres ejerzan (restrictivo, covisionado o técnico) está relacionado con un menor tiempo de conexión a internet en los hijos. 
Tabla 2

Correlación entre tipo de control de los padres percibido por los hijos y el tiempo de conexión de estos a internet.

\begin{tabular}{c|c|c|c|c|c|c} 
& \multicolumn{2}{|c|}{$\begin{array}{c}\text { Índice de } \\
\text { conectividad }\end{array}$} & \multicolumn{2}{c|}{$\begin{array}{c}\text { Índice de } \\
\text { adicción a internet }\end{array}$} & \multicolumn{2}{|c}{$\begin{array}{c}\text { Índice de } \\
\text { adicción al celular }\end{array}$} \\
& $\mathbf{N}$ & $\mathbf{R h o}$ & $\mathbf{N}$ & $\mathbf{R h o}$ & $\mathbf{N}$ & $\mathbf{R h o}$ \\
\hline Conductual materno & 155 & -.066 & 210 & -.024 & 210 & -.092 \\
Conductual paterno & 126 & $-.282^{* * *}$ & 171 & -.142 & 171 & $-.183^{*}$ \\
$\begin{array}{c}\text { Psicológico } \\
\text { materno }\end{array}$ & 160 & .140 & 213 & $.222^{* * *}$ & 213 & $.279^{* * *}$ \\
Psicológico paterno & 131 & $-.306^{* * *}$ & 174 & -.018 & 174 & -.110 \\
Control restrictivo & 223 & $-.334^{* * *}$ & 233 & .071 & 233 & $-.218^{* * *}$ \\
Control covisionado & 222 & $-.347^{* * *}$ & 232 & $-.161^{*}$ & 232 & $-.201^{* *}$ \\
Control técnico & 180 & $-.243^{* * *}$ & 189 & -.049 & 189 & $-.151^{*}$
\end{tabular}

${ }^{*} p \leq .05 ;{ }^{* *} p \leq .01 ;{ }^{* * *} p \leq .001$.

Fuente: elaboración propia

En relación con las conductas adictivas (Tabla 2), se puede observar que el control psicológico materno presenta correlación positiva tanto con la adicción a internet (Rho $=.222)$ como con la adicción al celular (Rho $=.279)$. Es decir, que a mayor control psicológico por parte de la madre, mayor será la presencia de conductas adictivas a internet y al celular en los hijos. Es importante recordar que este tipo de control (psicológico) es un control coercitivo, que induce a la culpa, recurriendo a la crítica excesiva y limitación de la autonomía. También es posible observar que ejercer cualquier tipo de control por parte de los padres sobre el uso de internet disminuye la adicción al celular, mientras que se observa una relación negativa baja entre el control covisionado y la adicción a internet $($ Rho $=$ -.161) y el control conductual paterno con la adicción al celular (Rho $=-.183)$.

Al examinar las conductas de riesgo en los participantes y su relación con el control ejercido por sus padres, como lo evidencia la Tabla 3, es el control psicológico materno el que presenta alguna relación directa, tanto con la presencia de conductas de riesgo en los hijos (Rho $=.293$ ) como con los riesgos que estos asumen en el uso de internet $(R h o=$ .194). Esto indica que a mayor control psicológico de la madre, más conductas de riesgo asumen los hijos, e incluso, si por el contrario, la madre ejerce un mayor control conductual, menor será la presencia de conductas de riesgo en el hijo (Rho $=-.172)$. 
Tabla 3

Correlación entre tipo de control y de mediación de los padres percibido por los hijos y sus conductas de riesgo.

\begin{tabular}{c|c|c|c|c}
\multirow{2}{*}{ Tipo de control } & \multicolumn{2}{|c|}{ Conductas de riesgo } & \multicolumn{2}{c}{ Riesgos en internet } \\
& $\mathbf{N}$ & $\mathbf{R h o}$ & $\mathbf{N}$ & Rho \\
\hline Conductual materno & 210 & $-.172^{*}$ & 206 & .032 \\
Conductual paterno & 171 & -.143 & 170 & -.148 \\
Psicológico materno & 213 & $.293^{* * *}$ & 210 & $.194^{* *}$ \\
Psicológico paterno & 174 & .067 & 173 & -.121 \\
Control restrictivo & 233 & $-.212^{* * *}$ & 227 & $-.163^{*}$ \\
Control covisionado & 232 & $-.158^{*}$ & 228 & -.104 \\
Control técnico & 188 & -.020 & 184 & .028 \\
Mediación activa & 209 & $-.162^{*}$ & 206 & -.078 \\
Mediación restrictiva & 210 & $-.204^{* *}$ & 207 & $-.309^{* * *}$ \\
Mediación técnica & 204 & -.001 & 202 & -.110 \\
Monitoreo & 208 & -.116 & 205 & -.091 \\
& & & &
\end{tabular}

${ }^{*} \mathrm{p} \leq .05 ;^{* *} \mathrm{p} \leq .01 ;{ }^{* * *} \mathrm{p} \leq .001$.

Fuente: elaboración propia

Por otro lado, es el control restrictivo frente al uso de internet el que tiene mayor relación con una menor presencia de conductas de riesgo en general $(R h o=-.212)$ y menores riesgos asumidos por los hijos en internet (Rho $=-.163$ ). También el control covisionado tiene una relación baja negativa con la presencia de conductas de riesgo en general $($ Rho $=-.158)$ (Tabla 3).

También se evaluó la relación de las conductas de riesgo reportadas por los NNA con el tipo de mediación que sus padres dijeron ejercer frente al uso de internet, encontrando que, a mayor mediación restrictiva, menor presencia de conductas de riesgo en general $(R h o=-.204)$ y menores riesgos asumidos por sus hijos en el uso de internet $($ Rho $=-.309)$. También se evidenció una correlación baja negativa entre la mediación activa y la presencia de conductas de riesgo $($ Rho $=-.162)$.

Se consideró pertinente indagar por la relación entre las habilidades digitales reportadas por los padres y el tipo de mediación que ellos mismos dicen ejercer respecto al uso de internet por parte de sus hijos (Tabla 4), y se encontró una relación directa con la mediación activa $(R h o=.350)$ y técnica $(R h o=.265)$, así como con las actividades de monitoreo (Rho $=.360)$. Sin embargo, no se encontró relación entre las habilidades digitales de los padres y la mediación restrictiva. 
Tabla 4

Correlación entre tipo de mediación que reportan los padres y sus habilidades digitales en internet.

\begin{tabular}{c|c|c} 
& \multicolumn{2}{|c}{ Habilidades digitales de los padres } \\
Tipo de mediación & $\mathrm{N}$ & Rho \\
\hline Mediación activa & 208 & $.350^{* * *}$ \\
Mediación restrictiva & 209 & -.004 \\
Mediación técnica & 204 & $.265^{* * *}$ \\
Monitoreo & 207 & $.360^{* * *}$
\end{tabular}

${ }^{* * *} \mathrm{p} \leq .001$.

Fuente: elaboración propia

Finalmente, se indagó por la relación entre el tipo de control percibido por los hijos y el tipo de mediación reportada por los padres frente al uso de internet y, como lo muestra la tabla 5, si bien la correlación más alta está entre la mediación y el control restrictivos $($ Rho $=.384)$, algunas restricciones reportadas por los padres son valoradas como control covisionado $($ Rho $=.303)$ o control técnico $(R h o=.230)$ por sus hijos. También, mientras se evidencia una relación positiva entre la mediación activa y el control covisionado $(R h o=.334)$, al parecer, las acciones de dicha mediación también son percibidos por los hijos como control restrictivo $(R h o=.218)$.

Tabla 5

Correlación entre tipo de mediación que reportan los padres y el tipo de control percibido por los hijos.

\begin{tabular}{c|c|c|c|c}
\multirow{2}{*}{ Tipo de mediación } & \multicolumn{2}{|c|}{ Control covisionado } & Control restrictivo & Control técnico \\
& $\mathrm{N}$ & Rho & Rho & Rho \\
\hline Mediación activa & 194 & $.334^{* * *}$ & $.218^{* *}$ & .149 \\
Mediación restrictiva & 195 & $.303^{* * *}$ & $.384^{* * *}$ & $.230^{* *}$ \\
Mediación técnica & 190 & .037 & .134 & .133
\end{tabular}

${ }^{* *} p \leq .01 ;^{* * *} p \leq .01$.

Fuente: elaboración propia

\section{Discusión}

Esta investigación tuvo como objetivo identificar la relación de los controles y las mediaciones parentales con el uso de internet por niños, niñas y adolescentes colombianos.
De acuerdo con los resultados fue posible confirmarlas hipótesis planteadas sobre la relación entre el tipo de control de los padres tanto con el tiempo de conexión de los estudiantes a internet, como con sus conductas de riesgo y, además, que existe relación entre el tipo de mediación que reportan los padres y 
sus habilidades digitales en internet, así como la relación entre la mediación de los padres y el tipo de control percibido por los hijos.

Los resultados evidenciaron que cualquier tipo de control que los padres ejerzan sobre el uso de internet (restrictivo, covisionado o técnico) está relacionado con un menor tiempo de conexión de los hijos y una menor adicción al celular; y estos hallazgos coinciden con lo reportado por Uhls y Robb (2017) y Chang et al. (2018). Por otro lado, una relación negativa con la madre incrementa la presencia de conductas adictivas a internet y al celular en los hijos, así como la presencia de conductas de riesgo, sobre todo cuando el tipo de control que la madre ejerce es psicológico. Pero si la madre ejerce un mayor control conductual, menor será la presencia de conductas de riesgo en el hijo. Esto estaría asociado con los hallazgos de Katz, Lemish, Cohen y Arden (2019) -respecto a estilos parentales controladores relacionados con niveles más elevados de involucramiento en cyberbullying por parte de los hijos, tanto como perpetradores y como víctimas-y lo encontrado por Álvarez-García, Núñez, García y Barreiro-Collazo (2018), quienes afirman que el control conductual, las reglas en el uso de internet y la monitorización en el uso de TIC son factores protectores de la ciberagresión.

Los resultados de esta investigación evidencian que tanto el control como la mediación restrictivos frente al uso de internet tienen relación con una menor presencia de conductas de riesgo en general y menores riesgos asumidos por los hijos en internet, lo que podría contradecir los hallazgos de Wright (2016) que relacionan la mediación parental restrictiva con el cyberbullying. También, algunos resultados coinciden con Chang et al. (2018), quienes concuerdan en que las estrategias de mediación restrictivas suelen ser factores protectores de la adicción al uso de TIC. Además, es necesario combinar la mediación restrictiva con acciones de mediación activa. Así, aunque baja, los resultados del presente estudio muestran una relación entre el control covisionado y la mediación activa con una menor adicción de los NNA a internet y una menor presencia de conductas de riesgo en general (Wright, 2016).

Por otro lado, los hallazgos también concuerdan con el estudio de Yubero, Larrañaga, Navarro y Elche (2018), al encontrar una relación directa entre las habilidades digitales de los padres, que puede equivaler a lo que los autores denominan como padres más activos en internet, con la mediación activa y técnica, así como con las actividades de monitoreo. Sin embargo, el $69.1 \%$ de los NNA entrevistados afirman saber más de internet que sus padres, por lo que tanto, el control técnico como la mediación técnica, es probable que sea poco utilizada por los padres $y$, por el contrario, recurran más frecuentemente a la restricción, e incluso, algunos intentos de participación de los padres en las actividades que sus hijos realizan en internet o en las TIC parece ser interpretada por estos como restricción o intrusión.

Acerca de las limitaciones del estudio es importante decir que, al haber utilizado una muestra por conveniencia, de tamaño limitado y con un rango de edad tan amplio, sus resultados no pueden ser generalizados a la totalidad de la población colombiana. Además, por tratarse de una investigación con diseño cuantitativo, no fue posible obtener datos complementarios relacionados con las experiencias sobre la mediación y uso de las TIC por parte de los participantes. Estas limitaciones sugieren realizar estudios futuros en los que se incluyan muestras estadísticamente representativas con diseños mixtos que incluyan estrategias de generación de datos cualitativos.

Esta investigación podría servir como base para el diseño e implementación de programas de formación psicoeducativa para padres, madres y cuidadores en general, encaminados al desarrollo de competencias y estrategias de mediación parental efectivas para el acompañamiento de los NNA en el uso que hacen de las tecnologías digitales. Además, 
los hallazgos de este estudio podrían sentar las bases científicas para la presentación de proyectos sociales que impacten las políticas públicas sobre el uso responsable y saludable de internet. Máxime, cuando Colombia cuenta con un Ministerio de las Tecnologías de la Información y la Comunicación y estrategias mediáticas de divulgación sobre el cuidado en el uso de las TIC.

Finalmente, las investigaciones que en psicología se han hecho sobre el tema en América Latina resultan limitadas. Por este motivo, los hallazgos de este trabajo constituyen un avance relevante sobre el tema en la región desde el punto de vista disciplinar. Con este artículo se busca alentar a los investigadores a continuar avanzando en el conocimiento que se tiene sobre las relaciones entre la mediación parental y el uso de las TIC por parte de los NNA.

\section{Referencias bibliográficas}

Alonso-Ruido, P., Rodríguez-Castro, Y., Lameiras-Fernández, M. y Martínez-Román, R. (2018). El sexting a través del discurso de adolescentes españoles. Saude e Sociedade, 27(2), 398-409. https://doi.org/10.1590/S010412902018171835

Álvarez-García, D., Núñez, J., García, T. y Barreiro-Collazo, T. (2018). Individual, Family, and Community Predictors of Cyber-aggression among Adolescents. The European Journal of Psychology Applied to Legal Context, 10(2), 79-88. https://doi.org/10.5093/ejpalc2018a8

Andrade, P. y Betancourt, D. (2008). Prácticas parentales: una medición integral. En S. Rivera-Aragón, R. Díaz-Loving, R. SánchezAragón e I. Reyes-Lagunes (Eds.), La Psicología Social en México (Vol. XII, pp. 561-565). México: AMEPSO.

Aponte, D., Castillo, P. y González, J. (2017). Prevalencia de adicción a internet y su relación con disfunción familiar en adolescentes. Revista Clínicade Medicina de Familia, 10(3), 179-186. http://scielo.isciii.es/scielo.php?script $=$ sci arttext\&pid=S1699-695X2017000300179\&ln- $\mathrm{g}=\mathrm{es} \& \mathrm{t} \operatorname{lng}=\mathrm{es}$.

Arnaiz, P., Cerezo, F., Giménez, A. y Maquilón, J. (2016). Conductas de ciberadicción y experiencias de cyberbullying entre adolescentes. Anales de Psicología, 32(3), 761-769. https:// doi.org/10.6018/analesps.32.3.217461

Bartau-Rojas, I., Aierbe-Barandiaran, A. y Oregui-González, E. (2018). Mediación parental del uso de internet en el alumnado de Primaria: creencias, estrategias y dificultades. Comunicar, 26(54), 71-79. https://doi. org/10.3916/C54-2018-07

Baumrind, D. (2005). Patterns of parental authority and adolescent autonomy. New Directions for Child \& Adolescent Development, (108), 61-69. https://doi.org/10.1002/cd.128

Chang, F. C., Chiu, C. H., Chen, P. H., Chiang, J. T., Miao, N. F., Chuang, H. Y. y Liu, S. (2018). Children's use of mobile devices, smartphone addiction and parental mediation in Taiwan. Computers in Human Behavior, 93, 25-32. https://doi.org/10.1016/j.chb.2018.11.048

Festl, R. y Gniewosz, G. (2018). Role of mothers' and fathers' internet parenting for family climate. Journal of Social and Personal Relationships, 36(6), 1764-1784. https://doi. org/10.1177/0265407518771753

Garmendia Larrañaga, M., Casado del Río, M. A. y Martínez, G. (2016). Parental mediation strategies in Spain: predicting factors for different strategie. Zer 20(39), 13-27. https:// doi.org/10.1387/zer.15513

Garmendia, M., Jiménez, E., Casado, M. y Mascheroni, G. (2016). Net Children Go Mobile: Riesgos y oportunidades en internet y el uso de dispositivos móviles entre menores españoles (2010-2015). Madrid: Red.es/Universidad del País Vasco/EuskalHerrikoUnibertsitatea.

Jiménez-Morales, M., Montaña, M. y Medina-Bravo, P. (2020). Uso infantil de dispositivos móviles: Influencia del nivel socioeducativo materno. Comunicar, 28(64), 21-28. https://doi.org/10.3916/C64-2020-02

Katz, I., Lemish, D., Cohen, R. y Arden, A. (2019). When parents are inconsistent: Parenting style and adolescents' involvement in cyberbullying. Journal of Adolescence, 74, 1-12. https://doi. 
org/10.1016/j.adolescence.2019.04.006

Livingstone, S. y Helsper, E. (2008). Parental mediation and children's internet use. Journal of Broadcasting \& Electronic Media, 52(4), 581-599. https://doi. org/10.1080/08838150802437396

Marín-Cortés, A., Hoyos de los Ríos, O. y Sierra, A. (2018). Factores de riesgo y factores protectores relacionados con el ciberbullying entre adolescentes: una revisión sistemática. Papeles del Psicólogo, 40(2), 109-124. https://doi. org/10.23923/pap.psicol2019.2899

Martínez de Morentin, J. I., Cortés, A. y Medrano, C. (2014). internet use and parental mediation: A cross-cultural study. Computer \& Education, 70, 212-221. https://doi.org/10.1016/j. compedu.2013.07.036

Moreno-Carmona, N. D. (2014). Factores familiares y psicosociales asociados a problemas internalizados $y$ externalizados en adolescentes colombianos (Tesis doctoral). Departamento de Psicología, Universidad Iberoamericana, México, D. F. http://biblioteca.uia. mx/F/ A1TF3LDGGSX5VFQYPET3E23V1BGGEA1FX6QVE4EPEII5U6DX95-38585?fun-

$\mathrm{c}=$ full-set-set\&set_number $=018092 \&$ set entry $=000001 \&$ format $=999$

Moreno Carmona, N. D. (Ed.) (2018). Adolescencia y Familia. La transformación de la familia colombiana y su incidencia en los adolescentes. Bogotá: Ediciones Universidad Cooperativa de Colombia.

Osorio-Tamayo, D. L. y Millán Otero, K. L. (2020). Adolescentes en internet, la mediación entre riesgos y oportunidades. Revista Colombiana de Ciencias Sociales, 11(1), 153-180. https://doi.org/10.21501/22161201.2979

Özgür, H. (2016). The relationship between internet parenting styles and internet usage of children and adolescents. Computers in Human Behavior, 60, 411-424. https://doi. org/10.1016/j.chb.2016.02.081

Pérez Sánchez, R. y Torres Fernández, D. (2020). Mediación adulta de la internet: un estudio cualitativo con adolescentes costarricenses. Revista Colombiana de Educación, 1(79), 39-60. https://doi.org/10.17227/rce.num79-

\section{5}

Pichardo, M., Fernández, M., García, M. y García, A. (2014). Uso de internet y su relación con el clima familiar y el bullying. International Journal of Developmental and Educational Psychology, 4(1), 245-253. https://doi. org/10.17060/ijodaep.2014.n1.v4.609

Suárez, P. y Vélez, M. (2018). El papel de la familia en el desarrollo social del niño: una mirada desde la afectividad, la comunicación familiar y estilos de educación parental. Revista Psicoespacios, 12(20), 173- 198, https://doi. org/10.25057/issn.2145-2776

Tavares, A., Falcke, D. y Pereira, C. (2019). Sexting en la adolescencia: percepciones de los padres. Ciencias Psicológicas, 13(1), 19-31. https://doi.org/0.22235/cp.v13i1.1806

Torrecillas-Lacave, T., Vázquez-Barrio, T. y Monteagudo Barandalla, L. (2017). Percepción de los padres sobre el empoderamiento digital de las familias en hogares hiperconectados. El profesional de la información, 26(1), 97-104. https://doi.org/10.3145/epi.2017.ene.10

Tourangeau, R. (1984). Cognitive science and survey methods: A cognitive perspective. En T. Jabine, M. Straf, J. Tanur y R. Tourangeau (Eds.), Cognitive aspects of survey methodology: Building a bridge between disciplines (pp. 73-100). Washington, DC: National Academy Press

Uhls, Y. y Robb, M. (2017). How Parents Mediate Children's Media Consumption. En F. Blumberg y P. Brooks, Cognitive Development in Digital Contexts (pp. 325-343). Los Ángeles: UCLA. https://doi.org/10.1016/B978-0-12809481-5.00016-X

Vargas-Rubilar, J. y Arán-Filippetti, V. (2014). Importancia de la parentalidad para el desarrollo cognitivo infantil: una revisión teórica. Revista Latinoamericana de Ciencias Sociales, Niñez y Juventud, 12(1), 171-186.

Warren, R. y Aloia, L. (2019). Parenting Style, Parental Stress, and Mediation of Children's Media Use. Western Journal of Communication, 83(4), 483-500. https://doi.org/10.1080/1 0570314.2019 .1582087

Willis, G. B. (2005). Cognitive Interviewing: $A$ 
Tool for Improving Questionnaire Design. Sage: Thousand Oaks, CA.

Wong, D. F. K., Zhuang, X. Y. y Ng, T. K. (2018). Is Parental Control Beneficial or Harmful to the Development of Young Children in Hong Kong? Journal of Child and Family Studies, 28(3), 831-838. https://doi.org/10.1007/ s10826-018-1301-3

Wright, M. (2016). The buffering effect of parental mediation in the relationship betweet adolescent's cyberbuyllying victimization and adjustment difficulties. Child Abuse Review, 25, 345-358. https://doi.org/10.1002/car.2448 Yubero, S., Larrañaga, E., Navarro, R. y Elche, M. (2018). Padres, hijos e internet. Socialización familiar de la red. Universitas Psychologica, 17(2), 1-13. https://doi.org/10.11144/javeriana. upsy17-2.phis

Recibido: 10 de diciembre de 2019 Aceptado: 5 de abril de 2021 